\title{
Crude Oil Recovery from Oily Sludge by using Liquefied Dimethyl Ether: A Comparison with Conventional Extraction Methods
}

Dong Zhang, ${ }^{a, \dagger}$, Ying Huang, ${ }^{a, *},{ }^{*}$, Kazuyuki Oshita ${ }^{b}$, Masaki Takaoka ${ }^{b}$, Meng Ying $^{a}$, Zifei Sun ${ }^{a}$, Changdong Sheng ${ }^{a}$

a. Solid Waste Treatment \& Resource Recycle Laboratory, Department of Environmental Science and Technology, School of Energy and Environment, Southeast University, Nanjing, Jiangsu 210096, People's Republic of China

b. Department of Environmental Engineering, Graduate School of Engineering, Kyoto University, Cluster C, Kyoto daigaku-Katsura, Nishikyo-ku, Kyoto 615-8540, Japan

$\dagger$ These authors contributed equally

*Corresponding author

Waste Treatment and Resource Recycling Laboratory,

Department of Environmental Science and Technology,

School of Energy and Environment,

Southeast University, Nanjing, 210096, China

Email: huangying@seu.edu.cn

Tel: $+86-152614306003$ 


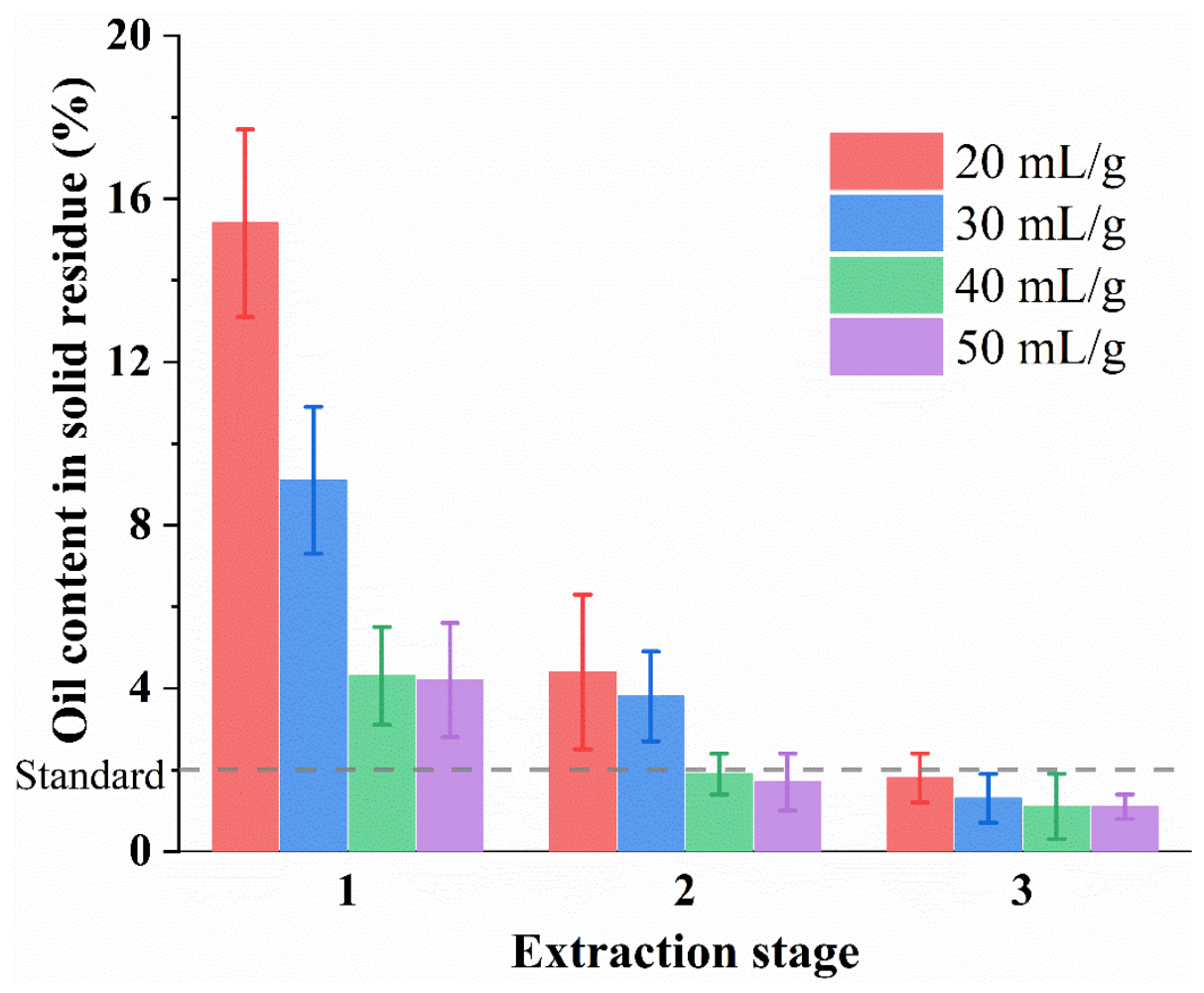

Figure S1. Oil content of solid residue by DME multiple extraction 


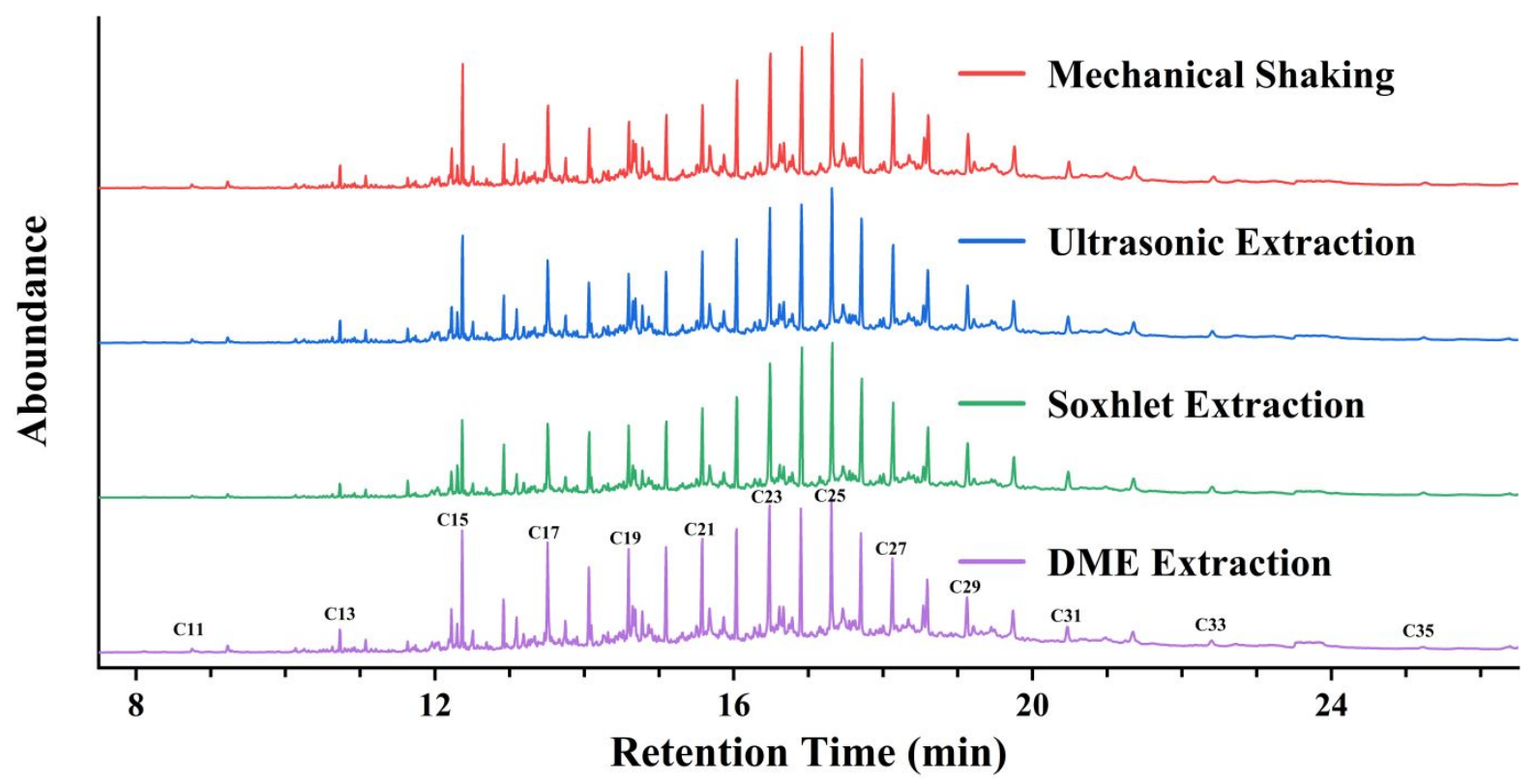

Figure S2. Gas chromatogram of saturates in the recovered oil, carbon numbers are noted 


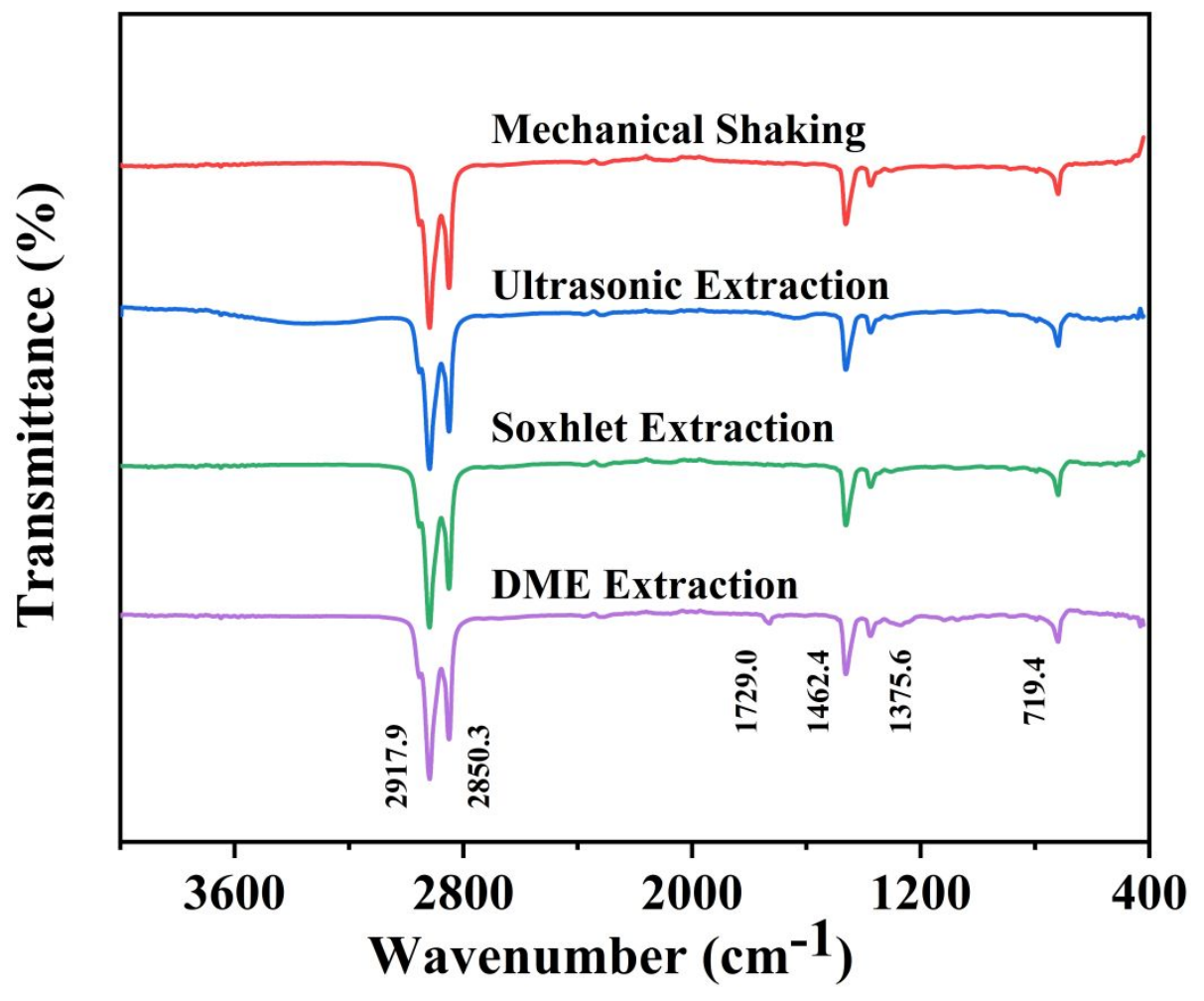

Figure S3. FTIR spectra of oil recovered by different extraction methods 